\title{
Multi-stage resection and repair for the treatment of adult giant sacrococcygeal teratoma: A case report and literature review
}

\author{
QIN-SONG SHENG, XIANG-MING XU, XIAO-BIN CHENG, WEI-BING WANG, \\ WEN-BIN CHEN, JIAN-JIANG LIN and JIA-HE XU
}

\begin{abstract}
Department of Colorectal and Anal Surgery, The First Affiliated Hospital of College of Medicine, Zhejiang University, Hangzhou, Zhejiang 310003, P.R. China
\end{abstract}

Received September 25, 2014; Accepted May 12, 2015

DOI: $10.3892 / \mathrm{ol} .2015 .3249$

\begin{abstract}
Sacrococcygeal teratoma (SCT) is a sacrococcygeal neoplasm derived from more than one primitive germ layer and is only occasionally encountered in adults. The primary treatment for all primary SCTs is surgical excision. The present study reports the case of a giant SCT in a middle-aged female with a history lasting $>3$ decades. Multi-staged surgical treatment was performed, including ileostomy plus tumor excision, four debridement plus flap repair procedures, and closure of the ileostomy. Follow-up showed improved quality of life without evidence of local recurrence after resection. The study also presents a brief overview of the relevant literature. To the best of our knowledge, this is the first report of multi-staged surgical treatment for giant SCT in an adult patient.
\end{abstract}

\section{Introduction}

Sacrococcygeal teratoma (SCT) is a neoplasm that arises in the sacrococcygeal region and is derived from multiple primitive germ layers (1). SCT is the most frequent congenital solid tumor encountered in fetuses and newborns, at a prevalence rate of 1 in 20,000-40,000 births (2) and with a female predominance of 3:1 (female:male) $(3,4)$. The most accepted pathogenetic mechanism of SCT is the overgrowth and migration of primitive node multipotent cells into the sacrococcygeal region (1). Other possible mechanisms include the non-sexual reproduction of germ cells in the gonads or in extragonadal sites, retention of 'wandering' germ cells over the migration of embryonic germ cells from the yolk sac to the gonads, and overproliferation of other multipotent embryonic cells (2). The majority of SCTs are cystic and benign, but $1-2 \%$ of cases are malignant and can be squamous cell carcinoma,

Correspondence to: Dr Jia-He Xu, Department of Colorectal and Anal Surgery, The First Affiliated Hospital of College of Medicine, Zhejiang University, 79 Qingchun Road, Hangzhou, Zhejiang 310003, P.R. China

E-mail: xujiahezju@163.com

Key words: sacrococcygeal teratoma, presacral tumor, presacral teratoma, teratoma, adult adenocarcinoma, sarcoma and other malignancies $(2,5)$. Use of ultrasonography, computed tomography, magnetic resonance imaging and needle biopsy allows the differential diagnosis of SCT prior to definitive surgery. Complete surgical resection remains the preferred treatment modality for SCT and shows a favorable prognosis in children and young adults (6).

The surgical treatment of older SCT patients is rarely reported in the current literature and the long-term treatment outcome remains unknown. Surgical resection of SCT is normally performed in a single stage; however, the present study describes multi-stage resection and repair in a middle-aged female who had suffered from a giant SCT from childhood, and also presents a literature review of adult SCT. To the best of our knowledge, this is the first report of multi-staged surgical treatment for giant SCT in an adult patient.

\section{Case report}

A 40-year-old female from a rural region presented to The First Affiliated Hospital of College of Medicine (December 15, 2011, Zhejiang University, Hangzhou, Zhejiang, China) with a 30-year history of a sacrococcygeal mass. The mass had progressively increased in size and became infected repeatedly. Standard medical care was not originally sought due to an underprivileged socioeconomic status. The patient's condition had significantly worsened within the last 2 years, with symptoms of recurrent high fever with spontaneous remission, but no involvement of the rectum, vagina or urethra. The past medical history and personal history were clinically insignificant.

Upon physical examination, a foul-smelling, pigmented and ulcerated mass was located in the sacrococcygeal region, with a size of $25 \times 15 \mathrm{~cm}$ (Fig. 1). Digital rectal examination identified a rigid irregular mass palpable behind the posterior rectal wall, while a transvaginal pelvic examination revealed negative signs. Hematological testing showed leukocytosis with a white blood cell count of $18 \times 10^{9} / 1$ (normal range, $4-10 \times 10^{9} / 1$ ) and a neutrophil percentage of $90 \%$ (normal range, 50-70\%). Other laboratory examination results, including clinical biochemistry and serum tumor biomarkers, such as $\alpha$-fetoprotein, carcinogenic embryonic antigen and neuron specific enolase, were within normal limits. Sacrococcygeal magnetic 
Table I. Multi-staged resection and repair of a huge sacrococcygeal teratoma.

\begin{tabular}{lllccc}
\hline Stage & Time-point & \multicolumn{1}{c}{ Surgery } & Surgical duration, min & Blood loss, ml & Blood transfusion \\
\hline Primary & 0 & Ileostomy + tumor excision & 260 & 400 & None \\
Secondary & 1 week & Debridement + VSD & 210 & 300 & None \\
& 2 weeks & Debridement + VSD & 140 & 200 & None \\
& 1 months & Debridement + flap transfer & 270 & 400 & 4.5 units RBCs \\
& 2 months & Debridement + flap transfer & 180 & 200 & None \\
Tertiary & 18 months & Closure of ileostomy & 80 & 50 & None \\
\hline
\end{tabular}

VSD, vacuum sealing drainage; RBCs, red blood cells.

resonance imaging (MRI) revealed an abnormal, large, high signal intensity involving the sacrococcygeal bone and pelvic adipose space, suggesting suppurative inflammation (Fig. 2). A pre-operative diagnosis of a sacrococcygeal cyst with complicating hidradenitis suppurativa was made.

Once the patient provided written informed consent, definitive surgery was scheduled due to the presence of recurrent sepsis. Surgical treatment was divided into three stages (Table I). The primary stage included prophylactic defunctioning loop ileostomy in the right lower quadrant in case of post-operative rectal leak, and complete resection of the sacrococcygeal cyst containing the coccyx and part of the sacrum, with a 1-cm tumor resection margin and preservation of the anorectum (Fig. 3). During the surgery, the tumor was found to be extremely large, and to involve the coccyx and part of the sacrum. The wound surface bled easily due to the inflammation. Important adjacent structures (rectum, anus, anal sphincter and autonomic nerve) were adequately protected. The wound was left unclosed, and the sterile dressing was changed on alternating days. From 1 week post-primary stage surgery onwards, the second stage included two sessions of debridement plus vacuum sealing drainage and two sessions of debridement plus flap transfer. A free flap was harvested from the left thigh, and the donor site was resurfaced using a free scalp flap.

The patient was discharged 2 weeks after the last session of flap transfer and was followed up at plastic surgery outpatient clinics. The patient experienced two episodes of mild skin graft infection, which were resolved after dressing care and antimicrobial treatment. The sacrococcygeal wound healed well with minimal scar retraction and mild skin graft ulceration and pigmentation (Fig. 4). The third stage was performed 18 months after the primary surgery to close the ileostomy. The patient experienced frequent defecation, 4-6 times daily, within the first 3 months post-operatively, which was resolved upon supplementation with a high-fiber diet and continence care.

Histological analysis identified the presence of various types of mature and differentiated tissues derived from the ecto-, meso- and endodermal layers, consistent with the diagnosis of mature teratoma (Fig. 5). The patient had been followed up as scheduled at surgical outpatient clinics for 12 months until the time of drafting this manuscript. Follow-up anorectal manometry and defecography showed normal anal sphincter tone, and good anal and urinary continence. The patient

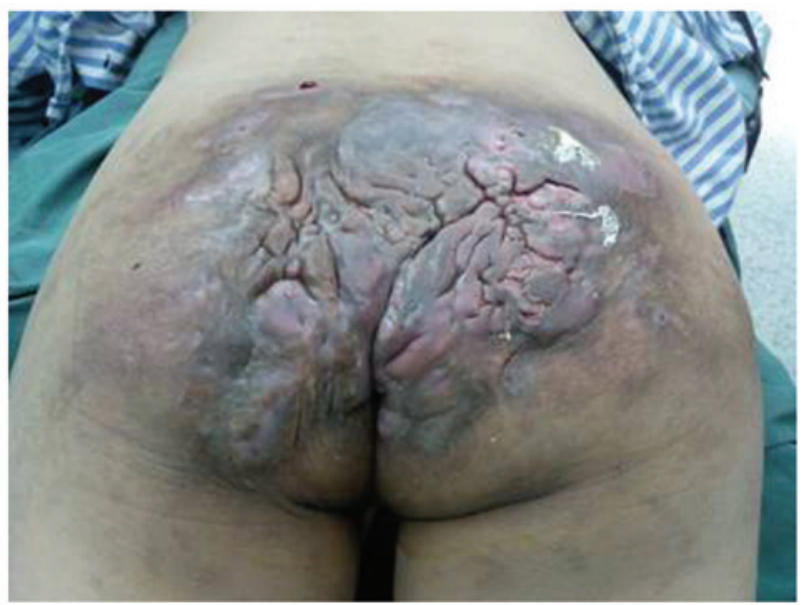

Figure 1. Gross appearance of the sacrococcygeal cyst.

complained of no clinically significant symptoms and/or signs and reported an improved quality of life without evidence of local recurrence after resection.

\section{Discussion}

Teratomas can occur in regions throughout the body, including the gonads, anterior mediastinum, retroperitoneal space and perineal area, but most frequently involve the sacrococcygeal region in infancy $(7,8)$. Adult SCT is only occasionally reported in the current literature, with fewer than 120 isolated cases (9). The Altman classification system is normally used to describe the anatomy of SCT relative to the body (10), as follows: Type I, predominantly external with a minimal presacral component, projecting from the sacrococcygeal region and distorting the buttocks; type II, predominantly external with a significant intrapelvic component; type III, predominantly intrapelvic with a small external buttock mass; and type IV, also called presacral teratoma, entirely internal without any external or buttock component. Type III is the most common type in adults (10), but in the present case, the patient had remained untreated since the initial occurrence as a teenager, and the SCT was classified as type II according to pre-operative MRI and intraoperative exploration.

Adult SCT may be asymptomatic or minimally symptomatic on the initial presentation and may be incidentally identified during routine physical or imaging examination in 


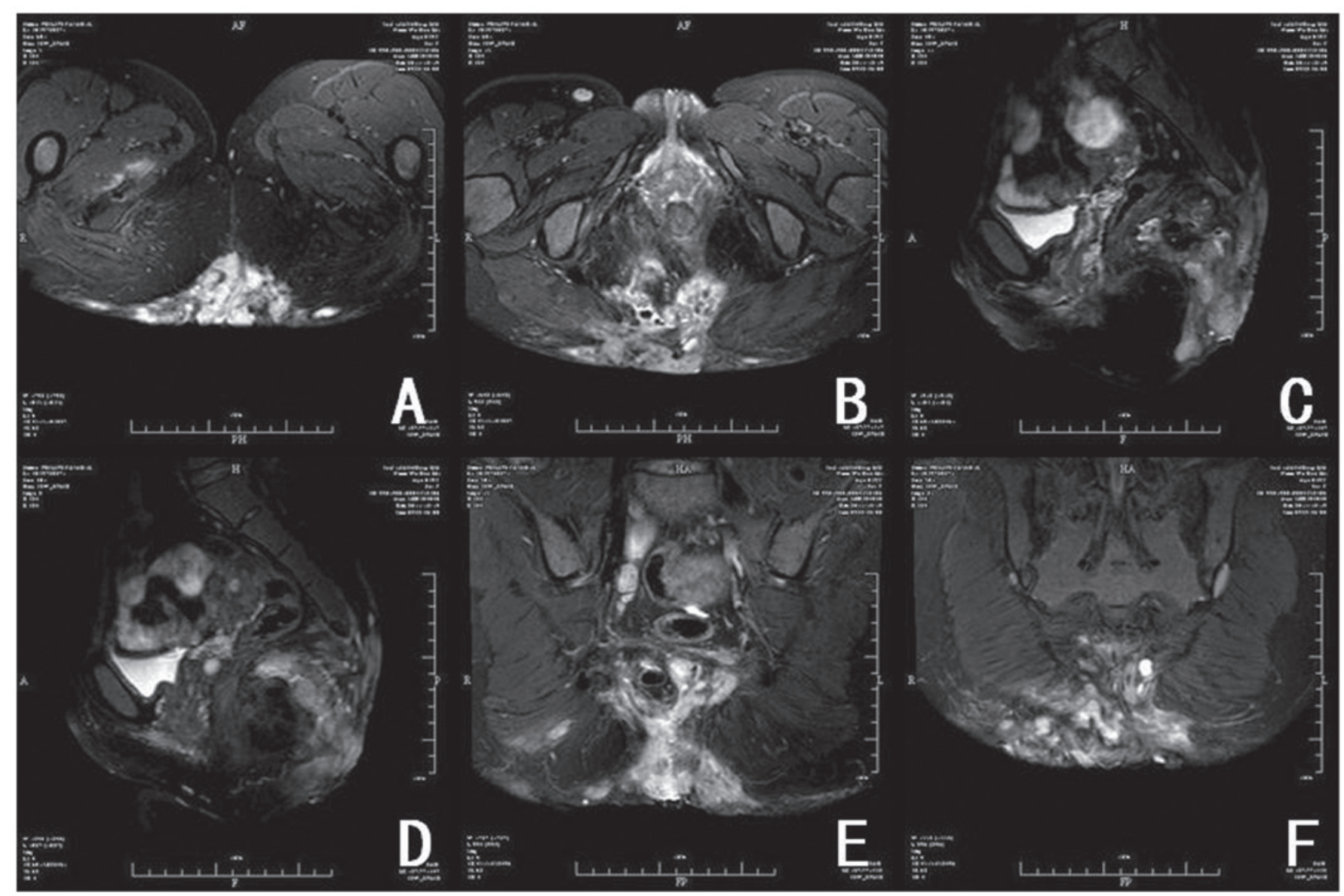

Figure 2. Magnetic resonance imaging scans of the sacrococcygeal cyst in the (A and B) transverse, (C and D) coronal and (E and F) sagittal views.

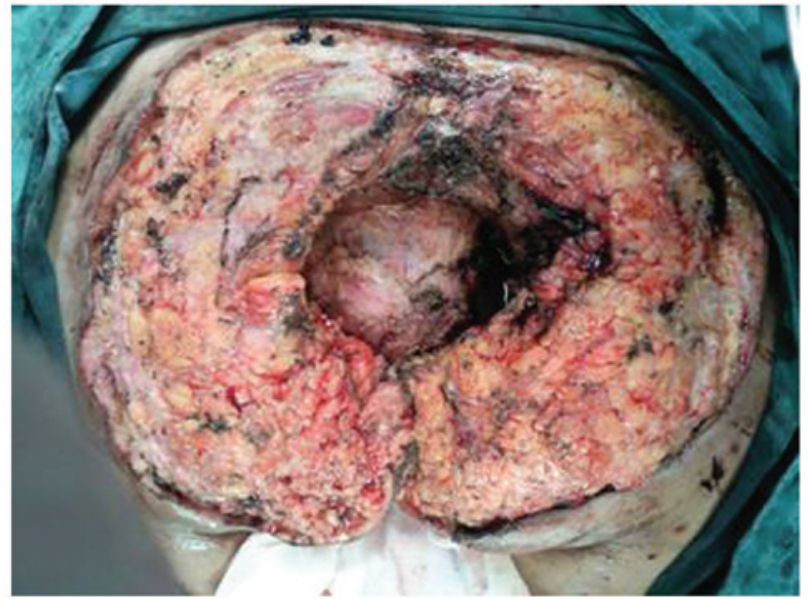

Figure 3. Complete excision of the sacrococcygeal teratoma, including the coccyx and part of the sacrum.

the majority of cases. SCT-associated symptoms depend on the size and location of the tumor, and include lower back pain, defecation or urinary difficulty, incontinence and venous engorgement of the lower limbs $(1,10)$. External compression and displacement of the vagina, uterus or rectum may also be found on pelvic and rectal examination $(1,11)$. If a complicating infection is present, SCT can present as a sacrococcygeal or perineal fistula or abscess. The SCT in the present case exhibited gradual predominant external growth, which resulted

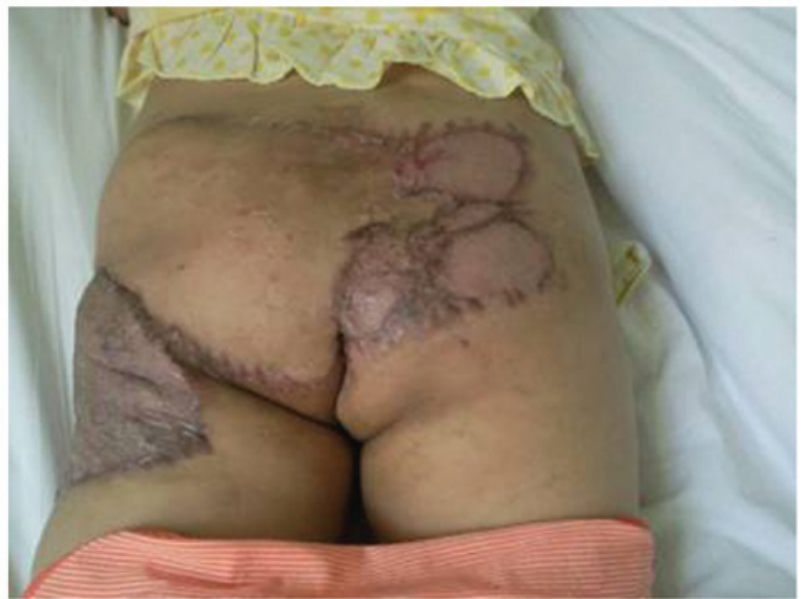

Figure 4. Physical appearance of the sacrococcygeal wound 6 months after the last session of flap transfer.

in roughening and pigmentation of the overlying skin (12). Complicating septic cellulitis and osteomyelitis caused a recurrent fever.

The evaluation of tumor biomarkers, such as $\alpha$-fetoprotein, carcinoembryonic antigen and human chorionic gonadotropin, is useful for the differential diagnosis of malignant teratoma and the surveillance of post-operative recurrence $(1,13)$. However, levels of all these markers were within the normal limits for the patient in the present case, consistent with a 


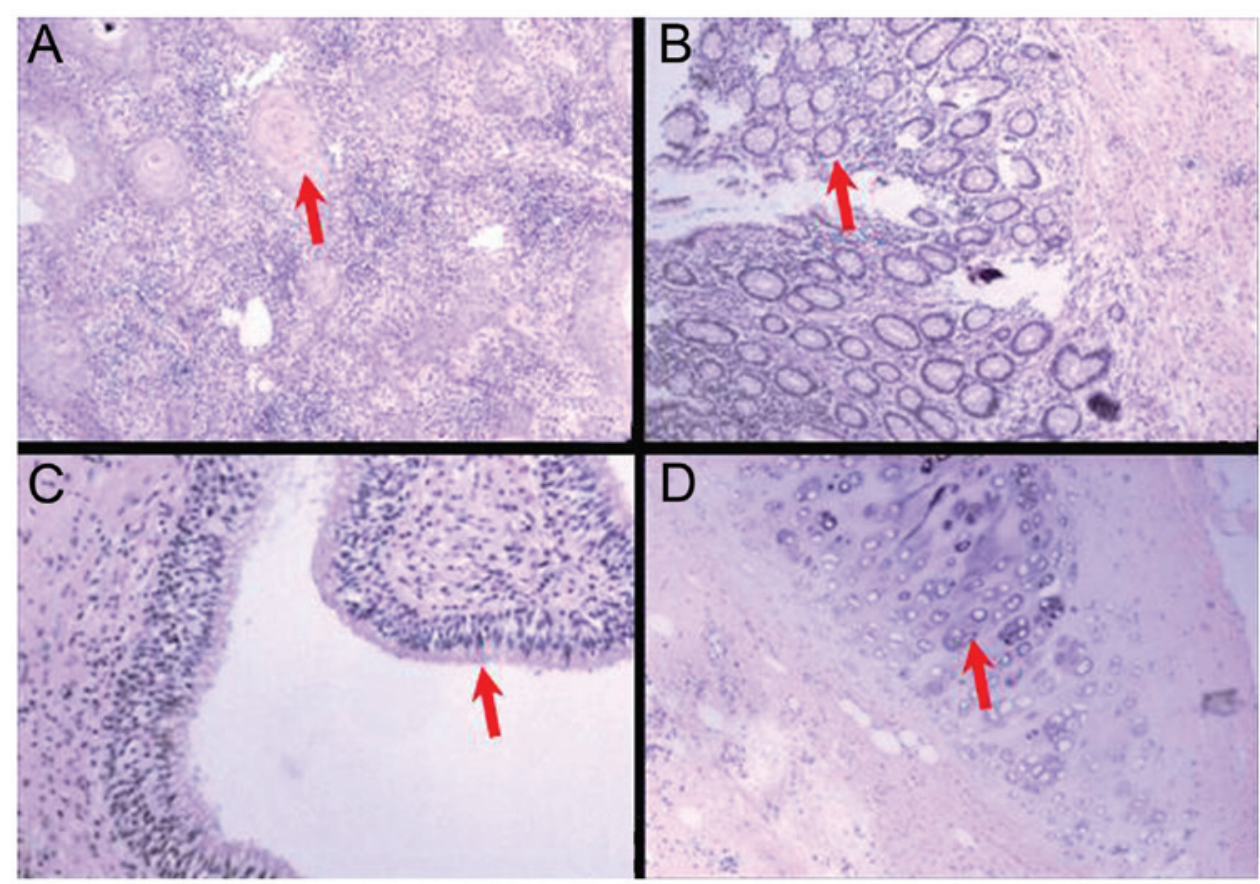

Figure 5. Histology (hematoxylin-eosin; magnification, x200) revealing a mature, differentiated teratoma containing all the three germ layers with complicating suppurative inflammation. The following cell types are indicated by arrows: (A) Clustered pyocytes, (B) glandular cells, (C) respiratory glandular cells and (D) chondrocytes.

30-year history and excluding the possibility of malignancy. Medical imaging examinations, including transvaginal and transrectal ultrasonography, computed tomography and MRI $(1,5)$, are useful for delineating the anatomical location and gross pathology, and for determining the optimal surgical approach (10). MRI is superior to computed tomography in terms of specificity and accuracy for visualizing a soft-tissue mass, such as SCT, containing abundant fat and fluid $(1,14)$. MRI in the present case revealed a large retrosacral soft-tissue mass involving the sacrococcygeal bone and accompanied by complicating suppurative inflammation. Needle biopsy, either transrectal or percutaneous, is seldom used for the pre-operative assessment of SCT due to the safety concerns of tumor cell seeding and puncture site infection (1), and as use of currently available medical imaging techniques can ensure an accurate and safe pre-operative assessment of SCT. However, Pendlimari et al (15) suggested that pre-operative biopsy may be useful in selected patients if neoadjuvant chemoradiation therapy is deemed beneficial and necessary. In the present case, the pre-operative evaluation revealed a localized sacrococcygeal soft-tissue mass with complicating septic inflammation, for which definitive complete excision was indicated. The differential diagnoses consisted of other uncommon adult benign or borderline malignant sacrococcygeal soft-tissue tumors or a congenital malformation, including neurofibroma, a giant cell tumor of the sacrum or a tailgut cyst $(1,2,7)$.

Complete surgical excision remains the mainstay definitive modality for the treatment of SCT. Early surgical intervention normally results in a favorable long-term treatment outcome and good quality of life. However, the definitive surgery was delayed in the present patient due to an underprivileged socioeconomic status. Multiple surgical approaches have been reported depending on the anatomical classification of
SCT, including an anterior abdominal approach, a retroanal approach, the combined access approach and the recently emerging laparoscopic-assisted approach (10). For SCT types II and III, the posterior trans-sacral approach with preservation of the rectum is used most often, while an additional abdominal incision may be required if the SCT extends into the pelvis and the retroperitoneal space to a certain extent (7). Concomitant excision of the coccyx is usually performed to eliminate the risk of tumor recurrence, which is reportedly $30-40 \%$ without coccygectomy $(2,9)$. Massive bleeding is the major surgical morbidity for the surgical excision of SCT; thus, meticulous dissection between the presacral fascia and the rectal fascia aids in the prevention of excessive blood loss and iatrogenic ureteral injuries (16). Other common surgical morbidities (31\%) include bladder dysfunction (15\%), fecal incontinence (7\%) and dysesthesia (7\%), as the nervous plexuses innervating the bladder and rectum, such as the pelvic splanchnic nerves, are frequently injured (16). Transcatheter arterial embolization may be useful for reducing blood loss during the excision of a large-sized tumor (2). In the present patient, complete excision of the SCT was performed with concomitant removal of the coccyx and part of the sacrum to minimize the risk of tumor recurrence. Moreover, a prophylactic ileostomy was performed to prevent rectal leakage and wound contamination. The patient underwent successful serial surgeries with minimal morbidities and remained free of recurrence at the final follow-up prior to preparation of this study.

Mature SCT has an extremely low potential for malignancy in adults, whereas immature teratoma, particularly that containing germ cell components, is likely to be malignant and carry a risk of recurrence. It remains unknown whether extended resection and adjuvant chemoradiation therapy are beneficial due to the rarity of malignant SCT and the lack 
of a standard management protocol (7). It is recommended that malignant SCT should be treated by a multidisciplinary surgical team at an advanced oncological center.

In conclusion, SCT more often occurs in newborns and is only rarely observed in adults, presenting as a gradually enlarging sacrococcygeal cystic mass. The diagnosis of adult SCT mainly depends on a combination of medical imaging examinations, particularly high-resolution MRI; however, the incorporation of other medical imaging techniques and tumor biomarker tests may aid in the differentiation and exclude the possibility of malignant SCT. Early complete excision is the preferred definitive modality of treatment for SCT, and multi-staged excision and reconstruction resulted in successful and safe treatment in the present case. Mature SCT is rarely malignant in adults, and close follow-up is essential for the detection of early recurrence.

\section{References}

1. Luk SY, Tsang YP, Chan TS, Lee TF and Leung KC: Sacrococcygeal teratoma in adults: case report and literature review. Hong Kong Med J 17: 417-420, 2011.

2. Afuwape OO, Ogundoyin OO, Ogunlana DI and Adeleye AO: Adult sacrococcygeal teratoma: A case report. Ghana Med J 43 : 40-42, 2009.

3. Goto S, Suzumori N, Obayashi S, Ozaki Y and Sugiura-Ogasawara M: Two cases of prenatally diagnosed sacrococcygeal teratoma type I with different clinical features. Congenit Anom (Kyoto) 53: 92-94, 2013.

4. Paramythiotis D, Papavramidis TS, Michalopoulos A, et al: Chronic constipation due to presacral teratoma in a 36-year-old woman: A case report. J Med Case Rep 4: 23, 2010.
5. Golas MM, Gunawan B, Raab BW, Füzesi L and Lange B: Malignant transformation of an untreated congenital sacrococcygeal teratoma: A amplification at $8 \mathrm{q}$ and $12 \mathrm{p}$ detected by comparative genomic hybridization. Cancer Genet Cytogenet 197: 95-98, 2010.

6. Okada T, Sasaki F, Cho K, et al: Management and outcome in prenatally diagnosed sacrococcygeal teratomas. Pediatr Int 50: 576-580, 2008

7. Ng EW, Porcu P and Loehrer PJ Sr: Sacrococcygeal teratoma in adults: Case reports and a review of the literature. Cancer 86 : 1198-1202, 1999.

8. Cho SH, Hong SC, Lee JH, et al: Total laparoscopic resection of primary large retroperitoneal teratoma resembling an ovarian tumor in an adult. J Minim Invasive Gynecol 15: 384-386, 2008.

9. Wishnia SC, Rosen JE, Hamid MA, Haas S and Moreno-Ruiz N: Management of a presacral teratoma in an adult. J Clin Oncol 26: 2586-2589, 2008.

10. Szyllo K and Lesnik N: Sacrococcygeal teratoma - case report and review of the literature. Am J Case Rep 14: 1-5, 2013.

11. Tsutsui A, Nakamura T, Mitomi H, et al: Successful laparoscopic resection of a sacrococcygeal teratoma in an adult: Report of a case. Surg Today 41: 572-575, 2011.

12. Roka YB, Koirala R, Bajracharya A, Shah S and Khaniya S: Giant sacrococcygeal teratoma in an adult: Case report. Br J Neurosurg 23: 628-629, 2009.

13. Hunter CJ, Ford HR, Estrada JJ and Stein JE: Alpha-fetoprotein levels correlate with the pathologic grade and surgical outcomes of pediatric retroperitoneal teratomas. Pediatr Surg Int 25: 331-336, 2009.

14. Lewis WT and Nyguen D: Radiological case submission: Mature presacral teratoma. Mil Med 174: 214-216, 2009.

15. Pendlimari R, Leonard D and Dozois EJ: Rare malignant neuroendocrine transformation of a presacral teratoma in patient with Currarino syndrome. Int J Colorectal Dis 25: 1383-1384, 2010.

16. Chen Y, Xu H, Li Y, et al: Laparoscopic resection of presacral teratomas. J Minim Invasive Gynecol 15: 649-651, 2008. 